\begin{tabular}{|c|l|}
\hline Title & Global existence of two-dimensional Navier-Stokes flow with nondecaying initial velocity \\
\hline Author(s) & Giga, Y.; Matsui, S.; Sawada, O. \\
\hline Citation & Hokkaido University Preprint Series in Mathematics, 503, 1-19 \\
\hline Issue Date & 2000-11-1 \\
\hline DOI & 10.14943/83649 \\
\hline Doc URL & http://hdl.handle.net/2115/69253 \\
\hline Type & bulletin (article) \\
\hline File Information & pre503.pdf \\
\hline
\end{tabular}

Instructions for use 


\section{Global Existence of}

\section{Two-Dimensional Navier-Stokes Flow}

with Nondecaying Initial Velocity

Y. Giga, S. Matsui and O. Sawada

Series \#503. November 2000 


\section{HOKKAIDO UNIVERSITY PREPRINT SERIES IN MATHEMATICS}

\#479 M.-H. Giga and Y. Giga, Crystalline and level set flow - Convergence of a crystalline algorithm for a general anisotropic curvature flow in the plane, 16 pages. 2000.

\#480 A. Arai and M. Hirokawa, Stability of ground states in sectors and its application to the Wigner-Weisskopf model, 16 pages. 2000.

\#481 T. Nakazi, Two dimensional $Q$-algebras, 11 pages. 2000.

\#482 N. H. Bingham and A. Inoue, Tauberian and Mercerian theorems for systems of kernels, 16 pages. 2000.

\#483 N. H. Bingham and A. Inoue, Abelian, Tauberian and Mercerian theorems for arithmetic sums, 29 pages. 2000 .

\#484 I. A. Bogaevski and G. Ishikawa, Lagrange mappings of the first open Whitney umbrella, 22 pages. 2000.

\#485 A. Arai and H. Kawano, A class of deformations of the Schrödinger representation of the Heisenberg commutation relation and exact solution to a Heisenberg equation and a Schrödinger equation, 22 pages. 2000 .

\#486 T. Nakazi, Functions in $N_{+}$with the positive real parts on the boundary, 21 pages. 2000.

\#487 Y. Shibukawa, Classification of the $R$-operator, 36 pages. 2000.

\#488 A. Inoue, Asymptotic behaviour for partial autocorrelation functions of fractional ARIMA processes, 20 pages. 2000.

\#489 S. Ohtani, Construction of unramified Galois extensions over maximal abelian extensions of algebraic number fields, 14 pages. 2000 .

\#490 T. Nakazi and T. Yamamoto, The real part of an outer function and a Helson-Szegö weight, 13 pages. 2000 .

\#491 A. Yamagami, On Gouvêás conjecture on controlling the conductor, 11 pages. 2000.

\#492 I. Tsuda and M. Hatakeyama, Making sense of internal logic: Theory and a case study, 10 pages. 2000.

\#493 I. Tsuda, Towards an interpretation of dynamic neural activity in terms of chaotic dynamical systems, 73 pages. 2000 .

\#494 T. Mikami, Optimal control for absolutely continuous stochastic processes and the mass transportation problem, 17 pages. 2000.

\#495 M. Arisawa and Y. Giga, Anisotropic curvature flow in a very thin domain, 21 pages. 2000.

\#496 T. Nakazi, Backward shift invariant subspaces in the bidisc, 9 pages. 2000.

\#497 Y. Giga, K. Inui, J. Kato and S. Matsui, Remarks on the uniqueness of bounded solutions of the NavierStokes equations, 4 pages. 2000.

\#498 Y. Giga, M. Paolini and P. Rybka, On the motion by singular interfacial energy, 21 pages. 2000.

\#499 J. Escher and Y. Giga, On a limiting motion and self-interactions of curves moved by the intermediate surface diffusion flow, 12 pages. 2000.

\#500 I. Tsuda and S. Kuroda, Cantor coding in the hippocampus, 20 pages. 2000.

\#501 M. Tsujii, Fat solenoidal attractors, 20 pages. 2000.

\#502 A. Arai, Ground state of the massless Nelson model without infrared cutoff in a non-Fock representation, 19 pages. 2000 . 


\title{
Global Existence of Two-Dimensional NavieR-Stokes Flow With Nondecaying Initial Velocity
}

\author{
Y. Giga, S. Matsui ${ }^{\dagger}$ and O. Sawada
}

Dedicated to Professor Takaaki Nishida and Professor Masayasu Mimura on their 60 th birthdays.

\begin{abstract}
A global-in-time unique smooth solution is constructed for the Cauchy problem of the Navier-Stokes equations in the plane when initial velocity field is merely bounded not necessary square-integrable. The proof is based on a uniform bound for the vorticity which is only valid for planar flows. The uniform bound for the vorticity yields a coarse globally-intime a priori estimate for the maximum norm of the velocity which is enough to extend a local solution. A global existence of solution for a $q$-th integrable initial velocity field is also established when $q>2$.
\end{abstract}

Matematics Subject Classification (1991). 35Q30, 76D05, 35K55.

Keywords. nondecaying initial velocity, two-dimentional Navier-Stokes flow, global existence, logarithmic Gronwall inequality, vorticity equation.

\section{Introduction}

We consider the nonstationary Navier-Stokes equations in the plane:

$$
\left\{\begin{array}{l}
u_{t}-\Delta u+(u, \nabla) u+\nabla p=0 \text { in }(0, T) \times \mathbb{R}^{2} \\
\operatorname{div} u=0 \text { in }(0, T) \times \mathbb{R}^{2} \\
\left.u\right|_{t=0}=u_{0} \quad\left(\text { with } \operatorname{div} u_{0}=0\right) \text { in } \mathbb{R}^{2}
\end{array}\right.
$$

where $u=u(t, x)=\left(u_{1}(t, x), u_{2}(t, x)\right)$ and $p=p(t, x)$ stand for the unknown velocity field of the fluid and its pressure field, while $u_{0}=u_{0}(x)=\left(u_{0}^{1}(x), u_{0}^{2}(x)\right)$

\footnotetext{
${ }^{*}$ Partly supported by the Grant-in-Aid for Scientific Reserch No. 10304010, the Japan Society for the Promotion of Science

†Partly supported by the Grant-in-Aid for Scientific Reserch No. 11640211, the Japan Society for the Promotion of Science.
} 
is a given initial velocity vector field; $x=\left(x_{1}, x_{2}\right)$ denotes a point of the plane $\mathbb{R}^{2}$ and $t(\geq 0)$ denotes the time.

Our goal is to prove the unique existence of global-in-time smooth solution of (NS) when initial data $u_{0}$ is merely bounded uniformly continuous, i.e., $u_{0} \in$ $B U C=B U C\left(\mathbb{R}^{2}\right)$ or more generally $u_{0} \in L^{\infty}\left(\mathbb{R}^{2}\right)$. (We do not distinguish spaces of vector-valued and scalar functions.) For a Banach space $X$ and an interval $I \subset \mathbb{R}$ let $C(I ; X)$ denote the space of all continuous functions on $I$ with values in $X$. We are now in position to state our main result.

Theorem 1. Assume that $u_{0} \in B U C$ satisfies div $u_{0}=0$ in $\mathbb{R}^{2}$ (in the sense of distribution). Then there exists a unique $u \in C([0, \infty) ; B U C)\left(\cap C^{\infty}((0, \infty) \times\right.$ $\left.\mathbb{R}^{2}\right)$ ) satisfying (NS) with $p=\sum_{i, j=1}^{2} R_{i} R_{j} u_{i} u_{j}$, where $R_{j}=(-\Delta)^{-1 / 2} \partial / \partial x_{j}$ is the Riesz operator.

Remark. If $u_{0} \in L^{\infty}\left(\mathbb{R}^{2}\right)$, it is known in [GIM] that there is a unique localin-time solution $u$ of (NS) in a suitable sense and $u(t) \in B U C\left(\mathbb{R}^{2}\right)$ for $t>0$. Thus by Theorem 1 it is extended to a global solution. We do not impose any smallness assumptions on $u_{0}$ in Theorem 1 .

There is a large literature on local existence of smooth solutions of (NS) even in a various domain of $\mathbb{R}^{n}(n \geq 2)$. It is also well-known that the solution can be extended globally in time provided that the initial velocity is small in various scaling invariant spaces. However, most of results assume a decay at space infinity for initial velocity. A recent paper of Amann [A] includes a nice survey of local solvability for initial data which decays at space infinity. The reader is referred to $[\mathrm{A}]$ for the state of arts. For nondecaying initial data there are only a few articles. Cannon and Knightly [CK] constructed a local solution which is continuous up to $t=0$ for bounded continuous initial data $u_{0}$ for $\mathbb{R}^{n}$. The method is based on the analysis developed by [K1]. Later it is extended in [K2] for bounded initial data. Local solvability for $u_{0} \in L^{\infty}$ is also mentioned in [C]. The method is based on Littlewood-Payley decomposition developed in [CM] 
and $[\mathrm{C}]$. More recently, K. Inui and the first two authors [GIM] constructed a local solution for $u_{0} \in B U C\left(\mathbb{R}^{n}\right)$ or $L^{\infty}\left(\mathbb{R}^{n}\right)$. Their key estimate can be written as

$$
\sup _{t>0} t^{1 / 2} \cdot\left\|\nabla E_{t}\right\|_{L^{1}\left(\mathbb{R}^{n}\right)}<\infty
$$

which yields the crucial estimate

$$
\sup _{0<t<1} t^{-1 / 2} \int_{0}^{t}\left\|\nabla E_{s}\right\|_{L^{1}\left(\mathbb{R}^{n}\right)} d s<\infty
$$

of [CK], where $E_{t}$ denotes the fundamental solution of the Stokes system $u_{t}-$ $\Delta u+\nabla p=0, \operatorname{div} u=0$ in $\mathbb{R}^{n}$. In [GIM] and [CK] the time $T_{0}$ where solution exists in $\left(0, T_{0}\right)$ is estimated by

$$
T_{0} \geq C /\left\|u_{0}\right\|_{\infty}^{2}
$$

with $C$ depending only on $n$. There are several novelty of [GIM] compared with [CK] or [C]. It proves that $u_{0} \in B U C\left(\mathbb{R}^{n}\right)$ implies $u(t, \cdot) \rightarrow u_{0}$ in $B U C$ as $t \rightarrow 0$ for the solution $u$. It also clarifies relation of solutions for the integral equation and the original equation (NS) and discusses the uniqueness of solutions. For nondecaying initial data relation $p=\sum_{i, j} R_{i} R_{j} u_{i} u_{j}$ is not automatically derived from the Poisson equation $-\Delta p=\operatorname{div}((u, \nabla) u)$, so it is included in our main statement so that the solution is unique. It is curious what condition of $p$ guarantees $p=\sum R_{i} R_{j} u_{i} u_{j}$. For this direction the reader is referred to a recent paper by J. Kato $[\mathrm{Ka}]$; see also [GIKM]. It seems that there are no results on solvability for exterior domains for nondecaying initial data $u_{0}$ although there is a large literature when $u_{0}$ is asymptotically constant at the space infinity and $n \geq 3$; see e.g. [BM, $\mathrm{KO}, \mathrm{S}]$.

It is well-known since Leray's pioneering work [L1] that there exists a global smooth solution if the initial data $u_{0}$ is in $L^{2}\left(\mathbb{R}^{2}\right)$, in other words, the initial kinematic energy is finite. For a such initial data the global existence of solution is proved by a priori estimate called an energy equality:

$$
\|u\|_{L^{2}}^{2}(t)+2 \int_{0}^{t}\|\nabla u\|_{L^{2}}^{2}(s) d s=\left\|u_{0}\right\|_{L^{2}}^{2}, \quad t>0
$$


This is formally obtained by multiplying $u$ with the first equation of (NS) and integrating by parts. Such an estimate is not expected for $u_{0} \in L^{\infty}\left(\mathbb{R}^{2}\right)$ so we develop a different a priori estimate for the $L^{\infty}$ norm $\|u\|_{\infty}(t)$ of the solution $u$.

Let us briefly explain main ideas in proving Theorem 1 .

(i) The local solution in [GIM] fulfills the integral equation

$$
u(t)=e^{t \Delta} u_{0}-\int_{0}^{t} \operatorname{div}\left(e^{(t-s) \Delta} \mathbb{P}(u(s) \otimes u(s))\right) d s,
$$

where $e^{t \Delta}$ denotes the heat semigroup and $\mathbb{P}$ denotes the Helmoltz projection and its $i j(1 \leq j, j \leq 2)$ component is of form $\delta_{i j}+R_{j} R_{j} ; \delta_{i j}$ denotes Kronecker's delta and $\otimes$ denotes tensor product; $\operatorname{div} F$ for tensor $F=\left(F_{i j}\right)_{i, j=1,2}$ is defined by a vector $\left(\sum_{j=1}^{2} \partial F_{i j} / \partial x_{j}\right)_{i=1,2}$.

(ii) As proved in [GIM] there is a regularizing effect so that $\nabla u(t) \in B U C$ for $t \in\left(0, T_{0}\right)$. Thus we may assume that $\nabla u_{0} \in B U C$ to prove the global existence.

(iii) In the plane the vorticity $\omega=\operatorname{rot} u=\partial u_{2} / \partial x_{1}-\partial u_{1} / \partial x_{2}$ is scalar and fulfills

$$
\omega_{t}-\Delta \omega+(u, \nabla) \omega=0
$$

(This equation is obtained by applying rot to the first equation of (NS).) The maximum principle yields that $\|\omega\|_{\infty}(t) \leq\left\|\omega_{0}\right\|_{\infty}$, where $\omega_{0}=\operatorname{rot} u_{0}$.

(iv) The crucial step is to establish

$$
\left\|\operatorname{div} e^{t \Delta} \mathbb{P}(f \otimes f)\right\|_{\infty} \leq C\left(1+\log R+\frac{1}{\sqrt{t}}\right)\|f\|_{\infty}\|\operatorname{rot} f\|_{\infty}+\frac{C}{R}\|f\|_{\infty}^{2}
$$

for all $R>1$ and all $t>0$, where $C$ is a constant independent of $t, f, R$. Using (1.4) to estimate the integral of (1.2) with $R=1+\|u\|_{\infty}(s)$, we obtain

$$
\begin{aligned}
\|u\|_{\infty}(t) \leq & \left\|u_{0}\right\|+C\left\|\omega_{0}\right\|_{\infty} \int_{0}^{t}(t-s)^{-1 / 2} \cdot\|u\|_{\infty}(s) d s+ \\
& +C\left(1+\left\|\omega_{0}\right\|_{\infty}\right) \int_{0}^{t}\left\{1+\log \left(1+\|u\|_{\infty}(s)\right)\right\} \cdot\|u\|_{\infty}(s) d s
\end{aligned}
$$

since $\|\omega\|_{\infty}(t) \leq\left\|\omega_{0}\right\|$. 
(v) In the similar way to prove the Gronwall inequality it turns out that (1.5) implies

$$
\|u\|_{\infty}(t) \leq K \exp \left(K e^{K t}\right)
$$

with $K>0$ depending only on $\left\|u_{0}\right\|_{\infty}$ and $\left\|\omega_{0}\right\|_{\infty}$. Although this estimate looks weak, because of (1.1) this yields the global solvability of (NS). To prove the inequality (1.4) we studies the derivatives of the Newton potentials carefully but the proof itself is not no complicated.

Since we do use the vorticity equation (1.3) it is not expected to generalize this method to the Dirichlet problem on an exterior domain or the half space instead of the whole space $\mathbb{R}^{2}$. If the boundary exists, the property $\|\omega\|_{\infty}(t) \leq$ $\left\|\omega_{0}\right\|_{\infty}$ is not expected since the vorticity is created near the boundary. The vorticity equation for $\mathbb{R}^{3}$ is

$$
\omega_{t}-\Delta \omega+(u, \nabla) \omega-(\omega, \nabla) u=0
$$

instead of (1.3). It is not expected to have $\|\omega\|_{\infty}(t) \leq\left\|\omega_{0}\right\|_{\infty}$ because of the vorticity streching term $(\omega, \nabla) u$ in $(1.7)$. Thus the present method is not expected to apply the three-dimensional setting. (In $\mathbb{R}^{3}$ the global existence of smooth solution for non small initial data is a famous open problem since Leray's pioneering work [L2] even for smooth $u_{0} \in L^{2}\left(\mathbb{R}^{3}\right)$.)

For $u_{0} \in L^{p}\left(\mathbb{R}^{2}\right), p>2$ we also prove that the global existence of smooth solution. The proof is easier than that of $B U C$ since $\mathbb{P}$ is bounded in $L^{q}\left(\mathbb{R}^{2}\right)$ for $1<q<\infty$ by the Calderón-Zygmund inequality. Since the local solution constructed by Amann [A] belongs to $L^{q}\left(\mathbb{R}^{2}\right)(q>2)$ for $t>0$, so his solution can be extended globally in time by our $L^{p}$ results. For $L^{2}\left(\mathbb{R}^{2}\right)$ initial data the global existence results goes back to Leray [L1]. For the initial data in the Lorentz space $L^{2, \infty}\left(\mathbb{R}^{2}\right)$ there is a unique global existence result [KY]; their proof is based on a kind of energy estimate.

The logarithmic type Gronwall inequality goes back to a work of Wolibner [W] which starts mathematical analysis on the Euler equation in $\mathbb{R}^{2}$. It is also found in a paper of Brezis and Gallouet [BG] for proving the global existence of 
solutions for some semilinear Schrödinger equation. However, in these papers the singular term $(t-s)^{-1 / 2}$ in (1.5) does not exist so the derivation of (1.6) is much easy. A similar argument to derive (1.5) from (1.4) by setting $R=$ $1+\|u\|_{\infty}$ is found in $[\mathrm{BG}]$.

This paper is organized as follows. In $\S 2$ we recall various properties of the Riesz operators to establish (1.4). In $\S 3$ we prove (1.6) by establishing the Gronwall type inequalities. We also prove Theorem 1 . In $\S 4$ we prove the global existence for $L^{q}$ initial data $(2<q<\infty)$.

After this work was completed, we were learned of a recent work of Koch and Tataru [KT] who study local well-posedness in the space $\mathrm{BMO}^{-1}\left(\mathbf{R}^{n}\right)$ of derivatives of $\mathrm{BMO}$ and related localized space $\mathrm{BMO}_{T}^{-1}$. Local existence for $L^{\infty}$ data is proved as a special element of $\mathrm{BMO}_{T}^{-1}$ for $T \in(0, \infty)$. However, for nonsmall initial data they do not discuss the global solvability for $\mathbb{R}^{2}$. The authors are grateful to Professor Herbert Koch for informing that [KT] is applicable for $L^{\infty}$ initial data and for pointing out [W].

After this work was completed, we were learned of a recent work of J. C. Mattingly and Ya G. Sinai [MS] who among other results gives a different elementary proof of global existence of smooth solutions for two dimensional Navier-Stokes equation with periodic boundary condition. They use the vorticity equation (1.3) instead of the energy estimate. However, their assumption does not include our setting. The authors are also grateful to Professor Alice Chang for letting them know a recent article [MS]. They are also grateful to Professor Tohru Ozawa for letting them know [BG]. They are also grateful to Professor Hideo Kozono for letting us know the state of arts on the exterior problems.

Before closing this introduction we prepare several notations. Let $e^{t \Delta}$ denote the heat semigroup defined by

$$
e^{t \Delta} f=G_{t} * f, G_{t}(x)=(4 \pi t)^{-1} \exp \left(-|x|^{2} / 4 t\right) \text { for } t>0 \text { and } x \in \mathbb{R}^{2}
$$

where $*$ denotes convolution of functions defined in $\mathbb{R}^{2}$. Let $\mathscr{S}=\mathscr{S}\left(\mathbb{R}^{2}\right)$ denote the space of rapidly decreasing functions in the sense of $\mathrm{L}$. Schwartz. The divergence is denoted also by $\nabla \cdot$, for example, $\nabla \cdot F$ for a tensor $F=\left(F_{i j}\right)_{i, j=1,2}$ 
is $\left(\sum_{j} \partial_{j} F_{i j}\right)_{i=1,2}$ where $\partial_{j}=\partial / \partial x_{j}$.

\section{Estimate of the quadratic term}

Let $R_{i}$ be the Riesz operator whose symbol is $\sqrt{-1} \xi_{i} /|\xi|(i=1,2)$ i.e., $R_{i}=$ $(-\Delta)^{-1 / 2} \partial_{i}$. Let $K$ denote the fundamental solution of the minus Laplacian $-\Delta$ in $\mathbb{R}^{2}$, i.e., $K(x)=(-1 / 2 \pi) \log |x|$.

We first summarize some properties of the Riesz operator and the fundamental solution of the Laplacian, which may be well-known.

Lemma 1. The following identities hold for all $\varphi \in \mathscr{S}\left(\mathbb{R}^{2}\right)$ and $i, j, k=1,2$.

(1) $\quad R_{i} R_{j} \varphi=$ p.v. $\left(\partial_{i} \partial_{j} K\right) * \varphi-\frac{\delta_{i j}}{2} \varphi$.

(2) $\quad R_{i} R_{j} \varphi=\partial_{i} K * \partial_{j} \varphi=\partial_{j} K * \partial_{i} \varphi$

(3) $\quad R_{i} R_{j} \partial_{k} \varphi=R_{k} R_{j} \partial_{i} \varphi=R_{i} R_{k} \partial_{j} \varphi$.

(4) $\quad\left(R_{1}^{2}+R_{2}^{2}\right) \varphi=-\varphi$.

For an integer $m \geq 1$ there exists a numerical constant $C_{m}>0$ such that

(5) $\left|D^{m} K(x)\right| \leq \frac{C_{m}}{|x|^{m}}$,

where $D^{m} f(x)$ denotes one of $m$-th derivatives of $f(x)$.

Definition. Let $\chi_{I N}(x), \chi_{M I D}^{R}(x)$ and $\chi_{O U T}^{R}(x)$ be characteristic functions of $\{0 \leq|x| \leq 1\},\{1 \leq|x| \leq R\}$ and $\{R \leq|x|\}$ respectively for $R>1$. For the fundermental solution $K(x)=(-1 / 2 \pi) \log |x|$ and $i=1,2$ we put

$$
J_{I N}^{i}=\chi_{I N} \cdot \partial_{i} K, \quad J_{M I D}^{i}=\chi_{M I D}^{R} \cdot \partial_{i} K, \quad J_{O U T}^{i}=\chi_{O U T}^{R} \cdot \partial_{i} K .
$$


By definition $\partial_{i} K=J_{I N}^{i}+J_{M I D}^{i}+J_{O U T}^{i}$. We also define a (vector valued) layer potential $\mathbb{L}_{i}^{r}(\varphi)=\left(L_{i j}^{r}(\varphi)\right)_{j}$ by

$$
L_{i j}^{r}(\varphi)(x)=\int_{|y|=r} \partial_{i} K(y) \varphi(x-y) \frac{y_{j}}{|y|} d S_{y} .
$$

We shall estimate $L^{1}$-norms of these functions.

Lemma 2. There exists a numerical constant $C>0$ such that following estimates are valid.

(0) $\left\|\mathbb{L}_{i}^{r}(\varphi)\right\|_{1} \leq C\|\varphi\|_{1}$,

(1) $\left\|J_{I N}^{i} *(\nabla \varphi)\right\|_{1} \leq C\|\nabla \varphi\|_{1}$,

(2) $\left\|J_{M I D}^{i} *(\nabla \varphi)\right\|_{1} \leq C(1+\log R)\|\varphi\|_{1}$,

(3) $\quad\left\|\nabla\left\{\left(\chi_{O U T}^{i} \partial_{i j}^{2} K\right) * \varphi\right\}\right\|_{1} \leq \frac{C}{R}\|\varphi\|_{1}$

for all $\varphi \in \mathscr{S}$, all $R>1$, all $r>0$ and $i, j=1,2$, where $\partial_{i j}^{2}=\partial_{i} \partial_{j}$.

Proof. The estimates (0) and (1) are obtained by the Young inequality since $\partial_{i} K$ is integrable on any disk by Lemma $1-(5)$.

(2) Integrating by parts we have

$$
\begin{aligned}
J_{M I D}^{i} *\left(\partial_{j} \varphi\right)(x) & =\int_{1 \leq|y| \leq R}\left(\partial_{i} K\right)(y) \cdot\left(-\partial / \partial y_{j}\right)\{\varphi(x-y)\} d y \\
& =\left(\chi_{M I D}^{R} \cdot \partial_{i j}^{2} K\right) * \varphi-L_{i j}^{R}(\varphi)+L_{i j}^{1}(\varphi) .
\end{aligned}
$$

Here we note that $\left\|\left(\chi_{M I D}^{R} \cdot \partial_{i j}^{2} K\right) * \varphi\right\|_{1} \leq\left\|\chi_{M I D}^{R} \cdot \partial_{i j}^{2} K\right\|_{1} \cdot\|\varphi\|_{1}$. Hence (0) and Lemma 1-(5) imply

$$
\left\|J_{M I D}^{i} *(\nabla \varphi)\right\|_{1} \leq C(1+\log R)\|\varphi\|_{1} .
$$

This shows (2).

(3) Since $\partial_{l}\left\{\left(\chi_{O U T}^{i} \partial_{i j}^{2} K\right) * \varphi\right\}=\left(\chi_{O U T}^{i} \partial_{i j}^{2} K\right) *\left(\partial_{l} \varphi\right)$ holds, we have

$$
\left(\chi_{O U T}^{i} \partial_{i j}^{2} K\right) *\left(\partial_{l} \varphi\right)=\left(\chi_{O U T}^{i} \partial_{i j l}^{3} K\right) * \varphi+\int_{|y|=R} \partial_{i j}^{2} K(y) \varphi(x-y) \frac{y_{l}}{|y|} d S_{y} .
$$


By the Young inequality and Lemma 1-(5) $L^{1}$ norm of the righthand side is estimated by $(C / R)\|\varphi\|_{1}$ with a positive numerical constant $C$. The proof is now complete.

Lemma 3. There exists a numerical constant $C$ such that

$$
\left\|\nabla \cdot e^{t \Delta} \mathbb{P}(f \otimes f)\right\|_{\infty} \leq C\left(1+\log R+\frac{1}{\sqrt{t}}\right)\|f\|_{\infty}\|\operatorname{rot} f\|_{\infty}+\frac{C}{R}\|f\|_{\infty}^{2}
$$

for all $R>1$, all $t>0$ and all $f \in C^{1}\left(\mathbb{R}^{2}\right)$ with $\operatorname{div} f=0$ in $\mathbb{R}^{2}$.

Proof. By the duality $L^{\infty}$-norm for $F \in \mathscr{S}^{\prime}$ is characterized by

$$
\|F\|_{\infty}=\sup \left\{|<F, \varphi>| ; \varphi \in \mathscr{S} \text { with }\|\varphi\|_{1}=1\right\},
$$

where $\langle\cdot, \cdot\rangle$ denotes the pairing of (vector-valued) distribution and test functions. Thus to estimate $\|F\|_{\infty}$ for $F \equiv \nabla \cdot e^{t \Delta} \mathbb{P}(f \otimes f) \in \mathscr{S}^{\prime}$ we shall estimate $\left\langle F, \varphi>\right.$ assuming that $\varphi \in \mathscr{S}$ satisfies $\|\varphi\|_{1}=1$.

If a vector field $f$ satisfies $\operatorname{div} f=0$, then the identity $\nabla \cdot f \otimes f=(f, \nabla) f$ holds. Furthermore we have

$$
(f, \nabla) f=\operatorname{rot} f \times f+\frac{1}{2} \nabla|f|^{2},
$$

when $\times$ denotes the exterior product in $\mathbb{R}^{3}$ and $f=\left(f^{1}, f^{2}\right)$ is regarded as a vector valued function $\left(f^{1}, f^{2}, 0\right)$. This identity holds for vector fields in $\mathbb{R}^{3}$.

Since $\left\langle\nabla|f|^{2}, \mathbb{P} e^{t \Delta} \varphi\right\rangle=0$,

$$
<F, \varphi>=\left(\operatorname{rot} f \times f, \mathbb{P} e^{t \Delta} \varphi\right),
$$

where $(\cdot, \cdot)$ is $L^{2}\left(\mathbb{R}^{2}\right)$ inner product. Here, we put $\phi=e^{t \Delta} \varphi \in \mathscr{S}$ for a fixed $t$, which satisfies $\|\phi\|_{1} \leq 1$ and $\|\nabla \phi\|_{1} \leq C / \sqrt{t}$ with a numerical constant $C>0$.

Since $\mathbb{P}=\left[\delta_{i j}+R_{i} R_{j}\right]$ i.e. the $i j$ component equals $\delta_{i j}+R_{i} R_{j}$, by Lemma 1-(2) we get

$$
\begin{aligned}
\mathbb{P} \phi & =E \phi+\left[\partial_{i} K * \partial_{j}\right]_{i j} \phi \\
& =\left(E+\left[J_{I N}^{i} * \partial_{j}\right]+\left[J_{M I D}^{i} * \partial_{j}\right]+\left[J_{O U T}^{i} * \partial_{j}\right]\right) \phi
\end{aligned}
$$

with $2 \times 2$ identity matrix $E$. We now estimate each term. By Lemma 2 the following estimates hold : 


$$
\begin{aligned}
& \|E \phi\|_{1} \leq\|\phi\|_{1} \leq 1, \\
& \left\|J_{I N}^{i} * \partial_{j} \phi\right\|_{1} \leq C\|\nabla \phi\|_{1} \leq C^{\prime} / \sqrt{t}, \\
& \left\|J_{M I D}^{i} * \partial_{j} \phi\right\|_{1} \leq C(1+\log R)\|\phi\|_{1} \leq C(1+\log R) .
\end{aligned}
$$

Combining these estimates yields

$$
\begin{aligned}
\mid\left(\operatorname{rot} f \times f,\left(E+\left[J_{I N}^{i} * \partial_{j}\right]+\left[J_{M I D}^{i} * \partial_{j}\right] \phi\right) \mid\right. \\
\leq C(1+\log R+1 / \sqrt{t})\|\operatorname{rot} f\|_{\infty}\|f\|_{\infty}
\end{aligned}
$$

for $t>0$ and $R>1$. Using the identity $\operatorname{rot} f \times f=\nabla \cdot\left(f \otimes f-(1 / 2)|f|^{2} E\right)$, we have

$$
\left(\operatorname{rot} f \times f, J_{O U T}^{i} * \partial_{j} \phi\right)=-\left(f \otimes f-\frac{1}{2}\left|f^{2}\right| E, \nabla\left(J_{O U T}^{i} * \partial_{j} \phi\right)\right) .
$$

Here by the integration by parts we obtain $J_{O U T}^{i} * \partial_{j} \phi=\left(\chi_{O U T}^{R} \partial_{i j}^{2} K\right) * \phi+L_{i j}(\phi)$. From this identity we now observe that

$$
\nabla\left(J_{O U T}^{i} * \partial_{j} \phi\right)=\nabla\left(\chi_{O U T}^{R} \partial_{i j}^{2} K\right) * \phi+\nabla L_{i j}(\phi) \equiv \nabla F_{i j}+\nabla L_{i j}(\phi) .
$$

Thus

$$
\begin{aligned}
\left(\operatorname{rot} f \times f, J_{\text {OUT }}^{i} * \partial_{j} \phi\right)=-( & \left.f \otimes f-\left(\left|f^{2}\right| / 2\right) E, \nabla F_{i j}\right) \\
& -\left(f \otimes f-\left(\left|f^{2}\right| / 2\right) E, \nabla L_{i j}(\phi)\right) \\
=-( & \left.f \otimes f-\left(\left|f^{2}\right| / 2\right) E, \nabla F_{i j}\right) \\
& +\left(\operatorname{rot} f \times f, L_{i j}(\phi)\right) .
\end{aligned}
$$

Lemma 2-(0) and (3) implies $\left\|\nabla F_{i j}\right\|_{1}$ and $\left\|L_{i j}(\phi)\right\|_{1}$ are estimated by $C / R$ and $C$ respectively. This proves our lemma.

Since Lemmas 1 and 2 extend to $\mathbb{R}^{n}(n \geq 3)$, Lemma 3 also extends for $f \in C^{1}\left(\mathbf{R}^{n}\right)(n \geq 3)$ by interpreting rot $f$ in a suitable way.

\section{Logarithmic Gronwall inequality and a priori bounds}

In this section we derive a uniformly (in time) bound for a mild solution of (NS). For this purpose we establish the following logarithmic Gronwall inequality. 
Lemma 4. Let a nonnegative function $a(t, s)$ be continuous in $\{(t, s) \mid 0 \leq$ $s<t \leq T\}$ and satisfy $a(t, \cdot) \in L^{1}(0, t)$ for all $t \in(0, T]$ with some $T>0$. Assume that there exists a positive constant $\varepsilon_{0}$ and $A \in(0,1)$ such that

$$
\sup _{0 \leq t \leq T} \int_{t-\varepsilon_{0}}^{t} a(t, s) d s \leq 1-A .
$$

If positive constants $\alpha, \beta$ and a non negative function $f \in C([0, T])$ satisfy

$$
f(t) \leq \alpha+\int_{0}^{t} a(t, s) f(s) d s+\beta \int_{0}^{t}\{1+\log (1+f(s))\} \cdot f(s) d s
$$

for all $t \in[0, T]$. Then we have

$$
f(t) \leq\left\{\begin{array}{lc}
\frac{\alpha}{A} \cdot e^{\gamma t / A} & (\beta=0, a(t, s) \not \equiv 0) \\
-1+\frac{[(1+\alpha) e]^{\exp (\beta t)}}{e} & (\beta \geq 0, a(t, s) \equiv 0) \\
-1+\frac{[(1+\alpha / A) e]^{\exp (\beta+\gamma) t / A}}{e} & (\beta>0, a(t, s) \not \equiv 0)
\end{array}\right.
$$

for all $t \in[0, T]$. Here a positive constant $\gamma$ is defined by

$$
\gamma=\sup _{0 \leq t \leq T}\left\{\sup _{0 \leq s \leq t-\varepsilon_{0}} a(t, s)\right\}
$$

Remark. In the case of $a(t, s)=B(t-s)^{-\delta}$ with $0<\delta<1$ and $B>0$, it is easy to show that for any $A \in(0,1)$ there exists $\varepsilon_{0}$ of the form

$$
\varepsilon_{0}=\left(\frac{(1-\delta)(1-A)}{B}\right)^{1 /(1-\delta)} \text { so that } \gamma=B\left(\frac{(1-\delta)(1-A)}{B}\right)^{-\delta /(1-\delta)} \text {. }
$$

Proof. (i) The case $a(t, s) \equiv 0$ :

Let $F(t)$ be the right hand side of $(3.2)$ with $a(t, s) \equiv 0$. Computing $F^{\prime}(t)$ and using (3.2), we have

$$
\int_{0}^{t} \frac{F^{\prime}(s)}{\{1+\log (1+F(s))\}(1+F(s))} d s \leq \beta t
$$

We change the variable of integration by $y=1+\log (1+F(s))$ and integrate

$$
f(t) \leq F(t) \leq-1+\frac{[(1+\alpha) e]^{\exp (\beta t)}}{e}
$$


for all $t \in[0, T]$.

(ii) The case $a(t, s) \not \equiv 0$ :

The inequality (3.2) implies that $g(t)=\sup _{0 \leq \theta \leq t} f(\theta)$ satisfies

$$
\begin{gathered}
g(t) \leq \alpha+\int_{t-\varepsilon_{0}}^{t} a(t, s) d s \cdot g(t)+\int_{0}^{t-\varepsilon_{0}} a(t, s) g(s) d s+ \\
+\beta \int_{0}^{t}\{1+\log (1+g(s))\} \cdot g(s) d s \\
\leq \alpha+(1-A) \cdot g(t)+\int_{0}^{t-\varepsilon_{0}} \gamma g(s) d s+ \\
+\beta \int_{0}^{t}\{1+\log (1+g(s))\} \cdot g(s) d s \\
\leq \alpha+(1-A) \cdot g(t)+\int_{0}^{t}\{(\beta+\gamma)+\beta \log (1+g(s))\} \cdot g(s) d s .
\end{gathered}
$$

That is,

$$
g(t) \leq \frac{\alpha}{A}+\frac{1}{A} \cdot \int_{0}^{t}\{(\beta+\gamma)+\beta \log (1+g(s))\} \cdot g(s) d s .
$$

Applying (3.3) to this inequality with $\beta \neq 0$, we get our assertion because of $f(t) \leq g(t)$. In the case of $\beta=0$, it is easy to show our assertion by the standard way. This proves our lemma.

Now we state a priori estimate of solution for (NS). According to remarkes ( Step (v), (vi) ) in Introduction, the following estimate is enough to guarantee that the unique local-in-times solution obtained in [GIM] can be extended to a global solution. Thus we obtain Theorem 1.

Theorem 2. Let $u(t)$ be a mild solution of (NS), that is, $u(t)$ is a solution of the integral equation (1.2). Assume that $u$ and $\nabla u$ belong to $C\left(\left[t_{0}, t_{0}+T\right] ; B U C\right)$ for $T>0$ and $t_{0} \geq 0$. Then there exists a positive constant $K$ which depends only on $\left\|u\left(t_{0}\right)\right\|_{\infty}$ and $\left\|\operatorname{rot} u\left(t_{0}\right)\right\|_{\infty}$, such that

$$
\|u\|_{\infty}(t) \leq K \exp \left(K e^{K t}\right)
$$


for all $t \in\left[t_{0}, t_{0}+T\right]$.

Proof. We may of course assume that $t_{0}=0$ and $u\left(t_{0}\right)=u_{0}$.

By (1.2) we have

$$
\|u\|_{\infty}(t) \leq\left\|e^{t \Delta} u_{0}\right\|_{\infty}+\int_{0}^{t}\left\|\nabla \cdot\left(e^{(t-s) \Delta} \mathbb{P}(u(s) \otimes u(s))\right)\right\|_{\infty} d s .
$$

for $t \in[0, T]$. Applying Lemma 3 with $f=\|u\|_{\infty}(s)$ and $R=1+\|u\|_{\infty}(s)$ to this inequality, we have

$$
\begin{aligned}
\|u\|_{\infty}(t) \leq\left\|u_{0}\right\|_{\infty} & +\int_{0}^{t} C M \cdot(t-s)^{-1 / 2}\|u\|_{\infty}(s) d s+ \\
& +C(1+M) \cdot \int_{0}^{t}\left\{1+\log \left(1+\|u\|_{\infty}(s)\right)\right\} \cdot\|u\|_{\infty}(s) d s
\end{aligned}
$$

where $M=\sup _{0 \leq t \leq T}\|\operatorname{rot} u(t)\|_{\infty}$. On the other hand, $\omega(t)=\operatorname{rot} u(t)$ is a classical solution of the vorticity equation with the bounded coefficient $u \in$ $C\left([0, T] \times \mathbb{R}^{2}\right)$.

$$
\left\{\begin{array}{l}
\omega_{t}-\Delta \omega+(u, \nabla) \omega=0 \text { in }(0, T] \times \mathbb{R}^{2} \\
\omega(0)=\operatorname{rot} u_{0} \text { on } \mathbb{R}^{2}
\end{array}\right.
$$

Since the maximum principle $[\mathrm{PW}]$ yields $\sup _{0 \leq t \leq T}\|\omega\|_{\infty}(t) \leq\left\|\operatorname{rot} u_{0}\right\|_{\infty}$, the constant $M$ is estimated by $\left\|\operatorname{rot} u_{0}\right\|_{\infty}$. Thus by Lemma 4 and Remark after it imply our assertion.

The solution obtained in Theorem 1 is of cource equal to a local-in-time solution obtained in [GIM] for $0 \leq t \leq T_{0}$ for some $T_{0}>0$. Furthermore by the arguments in [GIM] $\left\|\operatorname{rot} u\left(t_{0}\right)\right\|_{\infty}$ with $0 \leq t_{0} \leq T_{0}$ is estimated by a quantity depending only on $\left\|u_{0}\right\|_{\infty}$ from above. Thus we have the following estimate.

Corollary 1. Let $u(t)$ be the unique solution of (NS) obtained in Theorem 1. Then there exists a positive constant $K_{0}$ depending only on $\left\|u_{0}\right\|_{\infty}$ such that

$$
\|u\|_{\infty}(t) \leq K_{0} \exp \left(K_{0} e^{K_{0} t}\right)
$$

for all $t \in[0, \infty)$. 


\section{$4 \quad L^{q}$ global estimate}

In this section we prove the following the global existence of (NS) for $L^{q}$ initial data with $2 \leq q<\infty$.

Theorem 3. Assume that $u_{0} \in L_{\sigma}^{q}\left(\mathbb{R}^{2}\right)$ for $2 \leq q<\infty$, where $L_{\sigma}^{q}\left(\mathbb{R}^{2}\right)$ is the solenoidal closed subspace of $L^{q}\left(\mathbb{R}^{2}\right)$. Then there exists a unique solution $u(t)$ of (NS), which belongs to $C\left([0, \infty) ; L_{\sigma}^{q}\left(\mathbb{R}^{2}\right)\right)$ as well as $C^{\infty}\left((0, \infty) \times \mathbb{R}^{2}\right)$.

Remark. Since $u(t)$ decays at space infinity in $L^{q}$-sense, the relation $p=$ $\sum_{i, j=1,2} R_{i} R_{j} u_{i} u_{j}$ is automatically fulfilled if $(u, \nabla p)$ is a solution of (NS).

For initial data $u_{0} \in L_{\sigma}^{q}\left(\mathbb{R}^{2}\right)$ the first author [G] obtained a unique localin-time mild solution $u(t)$ of (NS), which belongs to $C\left(\left[0, T_{0}\right) ; L_{\sigma}^{q}\left(\mathbb{R}^{2}\right)\right)$ and $C^{\infty}\left(\left(0, T_{0}\right) \times \mathbb{R}^{2}\right)$ with $2 \leq q<\infty$. Here $T_{0}$ satisfies $T_{0} \geq C\left\|u_{0}\right\|_{q}^{2 /(2 / q-1)}$ with a constant $C>0$ independent of $u_{0}$, where we denote the $L^{q}\left(\mathbb{R}^{2}\right)$ norm by $\|\cdot\|_{q}$. This solution, of cource, is a classsical soluion of (NS) in $(0, \infty) \times \mathbb{R}^{2}$. Thus as in the proof of Theorem 1, to prove Theorem 3 it is enough to show the following a priori estimate.

Theorem 4. Let $u(t)$ be a mild solution of (NS). Assume that $u$ and $\nabla u$ belong to $C\left(\left[t_{0}, t_{0}+T\right] ; L_{\sigma}^{q}\right)$ for $T>0, t_{0} \geq 0$ and $2 \leq q<\infty$. Then there exists a positive constant $C$ which depends only on $q$, such that

$$
\|u\|_{q}(t) \leq 2\left\|u\left(t_{0}\right)\right\|_{q} \exp \left(C\left\|\operatorname{rot} u\left(t_{0}\right)\right\|_{q} t\right)
$$

for all $t \in\left[t_{0}, t_{0}+T\right]$.

Proof. Without loss of generality, we may assume that $t_{0}=0$ and $u\left(t_{0}\right)=u_{0}$.

By (1.2) we have

$$
\|u\|_{q}(t) \leq\left\|e^{t \Delta} u_{0}\right\|_{q}+\int_{0}^{t}\left\|\nabla \cdot\left(e^{(t-s) \Delta} \mathbb{P}(u(s) \otimes u(s))\right)\right\|_{q} d s .
$$

for $t \in[0, T]$. It is well-known that the operator $\mathbb{P}$ is also a projection operator of $L_{\sigma}^{r}\left(\mathbb{R}^{2}\right)$ in $L^{r}\left(\mathbb{R}^{2}\right)$ for any $1<r<\infty$. Thus, applying Young's inequality and 
$\operatorname{div} u=0$ yields

$$
\begin{aligned}
\left\|\nabla \cdot\left(e^{(t-s) \Delta} \mathbb{P}(u(s) \otimes u(s))\right)\right\|_{q} & =\left\|\mathbb{P} e^{(t-s) \Delta}(u(s) \cdot \nabla) u(s)\right\|_{q} \\
& \leq C\left\|e^{(t-s) \Delta}(u(s) \cdot \nabla) u(s)\right\|_{q} \\
& \leq \frac{C}{(4 \pi(t-s))^{1 / q}} \cdot\|(u(s) \cdot \nabla) u(s)\|_{q / 2} \\
& \leq \frac{C}{(4 \pi(t-s))^{1 / q}} \cdot\|u(s)\|_{q} \cdot\|\nabla u(s)\|_{q}
\end{aligned}
$$

with some positive constant depending only on $q$. We now employ the CalderónZygmund inequality $\|\nabla f\|_{r} \leq C|| \operatorname{rot} f \|_{r}$ with a constant $C>0$ depending only on $1<r<\infty$ to get

$$
\|u\|_{q}(t) \leq\left\|u_{0}\right\|_{q}+\int_{0}^{t} \frac{C}{(t-s)^{1 / q}} \cdot\|u(s)\|_{q} \cdot\|\operatorname{rot} u\|_{q} d s .
$$

On the other hand, it is easy to see that $\|\operatorname{rot} u\|_{q}(t) \leq\left\|\operatorname{rot} u_{0}\right\|_{q}$ using the vorticity equation (see, eg. [GMO]). Hence we have

$$
\|u\|_{q}(t) \leq\left\|u_{0}\right\|_{q}+C\left\|\operatorname{rot} u_{0}\right\|_{q} \cdot \int_{0}^{t} \frac{1}{(t-s)^{1 / q}} \cdot\|u(s)\|_{q} d s .
$$

By the Gronwall inequality (Lemma 4 ) we have the desired a priori estimate.

Finally we give some remarks on the $n$ dimensional Navier-Stokes equations with $n \geq 3$;

$$
\left(\mathrm{NS}_{n}\right)\left\{\begin{array}{l}
u_{t}-\Delta u+(u, \nabla) u+\nabla p=0 \text { in }(0, T) \times \mathbb{R}^{n} \\
\operatorname{div} u=0 \text { in }(0, T) \times \mathbb{R}^{n} \\
\left.u\right|_{t=0}=u_{0} \text { in } \mathbb{R}^{n} .
\end{array}\right.
$$

In this case, a unique local-in-time solution which belongs to $C\left(\left[0, T_{0}\right] ; L^{q}\left(\mathbb{R}^{n}\right)\right)$ with $n<q \leq \infty$, has been obtained (see, eg. [GIM] and [G]). Note that $\nabla u \in C\left(\left(0, T_{0}\right] ; L^{q}\left(\mathbb{R}^{n}\right)\right)$ and $T_{0} \geq C /\left\|u_{0}\right\|_{q}^{2 /(-1+n / q)}$ with a positive constant depending only on $n$ and $q$. We state a criterion on a global solvabilty for the integral equation (1.2), whose solution is called a mild solution. 
Theorem 5. Assume that $n \geq 3$ and $n<q \leq \infty$. Let $u(t)$ be a mild solution $u \in C\left([0, T) ; L^{q}\left(\mathbb{R}^{n}\right)\right)$ of (NS). If there exists a positive constant $M$ such that

$$
\sup _{0 \leq t<T}\|\operatorname{rot} u\|_{q}(t) \leq M,
$$

then $u(t)$ can be extended beyond $T$ as a smooth solution with $u \in C\left([0, T] ; L^{q}\left(\mathbb{R}^{n}\right)\right)$ and $\nabla u \in C\left([0, T] ; L^{q}\left(\mathbb{R}^{n}\right)\right)$

Here in the case of $n \geq 4$ we define a vorticity (matrix) rot $u$ of a vector field $u=\left(u_{i}\right)_{i \downarrow}$ by

$$
\operatorname{rot} u \equiv \nabla u-{ }^{t} \nabla u
$$

where $\nabla u=\left(\partial_{j} u_{i}\right)_{i \downarrow, j \rightarrow}$ is a Jacobi matrix and ${ }^{t} \nabla u$ is its transposed matrix. Then it is easy to see

$$
(u, \nabla) u=(\nabla u) u=(\operatorname{rot} u) u+\left({ }^{t} \nabla u\right) u=(\operatorname{rot} u) u+\frac{1}{2} \nabla|u|^{2} .
$$

The proof of this theorem is along in the line for the proofs of Theorem 1 and 3 . So we safely omit the proof.

The result in Theorem 5 is by no means optimal but such a criterion on extendability is not completed included in the classical regularity criteria in the literature (e.g. [G]). 


\section{References}

[A] H. Amann, On the strong solvability of the Navier-Stokes equations, J. Math. Fluid Mech. 2(2000), 16-98.

[BM] W. Borchers and T. Miyakawa, On stability of exterior stationary Navier-Stokes flow, Acta. Math. 174(1995), 311-382.

[BG] H. Brezis and T. Gallouet, Nonlinear Schrödinger evolution equations, Nonlinear Analysis, TMA 4(1980), 677-681.

[CK] J. R. Cannon and G. H. Knightly, A note on the Cauchy problem for the Navier-Stokes equations, SIAM J. Appl. Math. 18 (1970), 641-644.

[C] M. Cannone, Ondelettes, Paraproduits et Navier-Stokes, Diderot Editeur, Arts et Sciences Paris-New York-Amsterdam (1995).

[CM] M. Cannone and Y. Meyer, Littlewood-Paley decomposition and NavierStokes equations, Methods and Applications of Analysis 2(1995), 307319 .

[G] Y. Giga, Solutions for semilinear parabolic equations in $L^{p}$ and regularity of weak solutions of the Navier-Stokes system, J. Differential Equations 61(1986), 186-212.

[GIM] Y. Giga, K. Inui and S. Matsui, On the Cauchy problem for the NavierStokes equations with nondecaying initial data, Quaderni di Matematica, 4(1999), 28-68.

[GIKM] Y. Giga, K. Inui, J. Kato and S. Matsui, Remarks on the uniqueness of bounded solutions of the Navier-Stokes equations, Hokkaido. Univ. Preprint Ser. in Math, \# 497, 2000.

[GMO] Y. Giga, T. Miyakawa and H. Osada, Two-dimentional Navier-Stokes flow with measures as initial vorticity, Arch. Rational Mech. Anal. 104(1988), 223-250. 
[Ka] J. Kato, On the uniqueness of nondecaying solutions of the Navier-Stokes equations, preprint.

[K1] G. H. Knightly, On a class of global solutions of the Navier- Stokes equations, Arch. Rational Mech. Anal. 21(1966), 211-245.

[K2] G. H. Knightly, A Cauchy problem for the Navier-Stokes equations in $\mathbf{R}^{n}$, SIAM J. Math. Anal. 3(1972), 506-511.

[KT] H. Koch and D. Tataru, Well-posedness for the Navier-Stokes equations, Adv. Math., to appear.

[KO] H. Kozono and T. Ogawa, On stability of Navier-Stokes flow in exterior domains, Arch. Rational Mech. Anal. 128(1994), 1-31.

[KY] H. Kozono and M. Yamazaki, Local and global unique solvability of the Navier-Stokes exterior problem with Cauchy data in the space $L^{n, \infty}$, Houston J. Math. 21(1995), 755-799.

[L1] J. Leray, Étude de diverses équations intégrales non linéaires et de quelques problèmes que pose l'Hydrodynamique, J. Math. Pures Appl. 12(1933), 1-82.

[L2] J. Leray, Sur le mouvement d'un liquide visquex emplissant l'espace, Acta Math. 63(1934), 193-248.

[MS] J. C. Mattingly and Ya G. Sinai, An elementary proof of the existence and uniqueness theorem for the Navier-Stokes equations, Math. Research Lett., to appear.

[PW] M. H. Protter and H. F. Weinberger, Maximum Principle in Differential Equations, Englewood Cliffs, N. J., Prentie-Hall, 1967.

[S] Y. Shibata, On an exterior initial-boundary value problem for the Navier-Stokes equations, Quart. Appl. Math. 57(1999), 117-155. 
[W] W. Wolibner, Un theorème sur l'existence du mouvement plan d'un fluide parfait, homogène, incompressible, pendent un temps infiniment long, Math. Z. 37(1933), 698-726.

Y. Giga

Department of Mathematics

Hokkaido University

Sapporo 060-0810, Japan

e-mail : giga@math.sci.hokudai.ac.jp

O. Sawada

Department of Mathematics

Hokkaido University

Sapporo 060-0810, Japan

e-mail : sawada@math.sci.hokudai.ac.jp
S. Matsui

Hokkaido Information University Ebetsu 069-8585, Japan

email : matsui@do-johodai.ac.jp 